\title{
Quarantine Exercises in the Time of Covid-19- A Review
}

\author{
Arti Sahu' ${ }^{1}$, Waqar M. Naqvi² \\ ${ }^{1}$ Department of Community Health Physiotherapy, Ravi Nair Physiotherapy College, Wardha, Maharashtra, India. \\ ${ }^{2}$ Department of Community Health Physiotherapy, Ravi Nair Physiotherapy College, Wardha, Maharashtra, India.
}

\section{ABSTRACT}

\section{BACKGROUND}

Coronavirus disease 2019 (Covid-19) originating in Wuhan, China in December 2019 spread worldwide rapidly. It was declared as a global public health emergency, and subsequently as a pandemic by the WHO, owing to its worldwide spread. Currently, Covid-19 is of the gravest concern because of its high transmissibility, high mortality, rapid global spread, lack of knowledge about the disease and lack of management resources. Lockdown and self-quarantine measures have been implemented globally by various nations to mitigate the spread of Covid-19. In several nations, fitness centres and other public places, where people are normally active, had been temporarily closed to prevent further spread of infection. Being quarantined at home for extended periods makes it challenging to stay physically active. Recent studies demonstrated that regular physical activities and exercises lower the incidence of several communicable diseases, including the novel Covid-19 by enhancing the immune system. Regular exercises improve the components of physical fitness, which include muscular strength, cardiorespiratory fitness, coordination, balance and agility, which in turn, enhance the physiological functions of the body. Regular exercises and relaxation techniques are some significant approaches aimed at improving one's overall health during the quarantine. Since organizations and governments worldwide had advised physical activity in quarantine, and also because regular physical activity is beneficial for both the physical and mental health, its role in the quarantine is significant. This review highlights the significance of physical activity and exercise in enhancing the well-being of individuals during the quarantine phase of Covid-19 pandemic.

\section{KEY WORDS}

Quarantine Exercises, Physical Activity, Quarantine, Pandemic, Covid-19

\author{
Corresponding Author: \\ Waqar M. Naqvi, \\ Professor and HOD, \\ Department of Community Health \\ Physiotherapy, \\ Ravi Nair Physiotherapy College, \\ Wardha, Maharashtra, India. \\ E-mail:waqar.naqvi@dmimsu.edu.in
}

DOI: $10.14260 / j e m d s / 2020 / 418$

Financial or Other Competing Interests: None.

How to Cite This Article:

Sahu A, Naqvi WM. Quarantine exercises in the time of covid-19- a review. J. Evolution Med. Dent. Sci. 2020;9(26):1922-1927, DOI: $10.14260 / \mathrm{jemds} / 2020 / 418$

Submission 17-04-2020,

Peer Review 02-06-2020,

Acceptance 09-06-2020,

Published 29-06-2020.

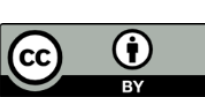




\section{BACKGROUND}

World Health Organization (WHO) declared the outbreak of the novel Coronavirus disease 2019 (Covid-19) as a Public Health Emergency of International Concern in January 2020. In March 2020, it was characterized as a pandemic,(1) Till 04/06/2020 WHO reported around 6,416,828 confirmed cases of Covid-19 and 382,867 deaths worldwide. The transmission of Covid-19 infection occurs via large droplets produced during coughing and sneezing by the infected patients. The infection usually occurs by inhaling these droplets but can also transmit through the aerosolization/ faecal-oral route.(2) People of all ages are at risk to the Covid19 infection. However, it shows a high mortality rate among the elderly, cancer patients, people with autoimmune diseases, people with HIV, people who underwent transplantation, and people with diabetes and/or hypertension. To minimize the contagion, health organizations requested social isolation in the form of quarantine. The major part of the activities was confined to the houses, leading to the forced closure of the establishments except those supplying basic needs. On March 21, 2020, the WHO European Committee formalized a letter within the institution's daily report, requesting that any physical activity should only be carried out in quarantine(3)

To prevent further spread of infection, organisations and governments in several countries have temporarily closed the fitness centres and other public places, where people are normally active. Being quarantined at home for extended periods can raise a major challenge in staying physically active.(4) The reduction in regular activity and exercise as a consequence of fear of infection as well as negative symptoms leads to a further compromise in the physical health and immunity of people.(5) Self-quarantine can contribute to anxiety and stress, thus challenging the mental wellbeing of people. Some significant approaches to improve one's physical and mental health during such a challenging condition are regular exercises and relaxation techniques. Among the measures adopted to contain the pandemic of Covid-19, is the closing of gyms and the limitation of the use of common spaces for physical exercise, consequently interrupting the training not only of athletes but also of nonathletes practising resistance training.(6) WHO recommends exercises of 150 minutes of moderate-intensity or 75 minutes of vigorous-intensity each week, or a combination of both. The given exercise proposal can easily be performed at home within limited space and without the requirement of special equipment.(4)

\section{Physical Inactivity and Sedentary Lifestyle during the Quarantine Period}

Amid Covid-19 pandemic, another pandemic has spread globally as the world seems to have adapted sedentary behaviour (SB) and physical inactivity (PI).(7) Since PI was declared a pandemic in 2012, major associations acknowledged this crisis and made attempts to enhance PA. However, there are still insufficient trends in physical activity. Several studies have demonstrated that even after the Covid-19 pandemic subsides, the health and economic impact of the SB pandemic will continue to be severe. SB has also been demonstrated to be an important forecaster of unfavourable health outcomes. Covid-19 precautionary measures leading to lockdown has accelerated the SB pandemic.(7) Due to lockdown measures, the public places have been temporarily shut down leading to discontinuation in a physically active lifestyle. However, several fitness centres have provided online exercise workout sessions to assist people in staying active and healthy at home. It is important to seriously consider the health risks associated with the current PI pandemic in the time of Covid-19.

Since a lot of people are working from home schedules due to quarantine, there has been a usual increase in the texting activity, video conferencing, hours of internet activity like online courses, webinars and all other important works are now done by relying on internet and technology. The increased use of handheld devices such as mobile phones, tablets, game controls, personal digital assistants and portable media players has led to an increase in the musculoskeletal problems during the quarantine period.(8) The studies reported that prolonged use of these devices can lead to constant mechanical stress on the musculoskeletal system, psychological strain, ergonomic risks leading to several musculoskeletal disorders, most commonly being neck pain. $(9,10)$ This lifestyle acquisition needs immediate attention as it can further lead to problems like postural impairment, text neck syndrome, nerve compression, muscle fatigue, spasm etc. which may further aggravate the musculoskeletal problems.

\section{How Physical Activities Play an Important Role during Quarantine?}

Physical activity (PA) and exercise play a key role in maintaining an appropriate health status by counteracting the detrimental impact of a variety of diseases, like diabetes mellitus, hypertension, cardiovascular diseases, respiratory diseases, or also to ensure active ageing in elderly by minimizing the chances of frailty, muscle loss, and dementia.(11) Exercises and physical activities have been proven effective in many chronic diseases, directly enhancing both physical and mental health. The primary reason to encourage regular exercise is to improve the components of physical fitness, which include muscular strength, cardiorespiratory fitness, coordination, balance and agility. These components have a direct relation with the physiological functions of the circulatory, respiratory, nervous, muscular and skeletal systems, and indirect relation with endocrine, immune, digestive, and/or renal systems.(11) Exercise is regarded as the true polypill, in regard to the epidemiological evidence of its preventive and therapeutic effects. Elderly require special attention in regard to the impact of PA and exercise in older people on many diseases, as well as supplemental effects on the characteristics of ageing and related disease conditions. (11)

Evidence shows that only normal, moderate and intense aerobic exercise improves the immune response to influenza vaccination relative to a sedentary lifestyle.(12) Long-term moderate aerobic exercise helps to decrease the risk of infection and enhance immune responses to respiratory infections.(12) Improving the basic physical activity regimen helps to improve safety and functional efficiency of the overall musculoskeletal system. Exercises augment several parameters by supplying nutrients to the musculoskeletal 
system, alleviating joint pain and rigidity, enhancing flexibility and mobility, and building muscle strength. Besides, they increase or preserve bone density, alleviate stress rates, release endorphins and enkephalins (to alleviate anxiety) and enhance overall body oxygenation. Thus, they produce a sense of well-being. Maintaining physical health is effective in preventing many health impacts with immediate effects on both physical and mental health. A specific prescription and guidance are required to ensure an effective exercise regime aimed at maintaining or enhancing the physical fitness' key health-related constituents.(13)

\section{Relationship between Physical Activities and Communicable Diseases \\ Epidemiological researches demonstrate the key role of routine physical activity and/or designed exercises in reducing the occurrence of several chronic diseases, which includes communicable diseases such as viral and bacterial infections, as well as non-communicable diseases such as cancer, diabetes, hypertension, and chronic inflammatory disorders. ${ }^{(14)}$ Exercise in itself reduces the chances of developing opportunistic infections, and it tends to improve the body's natural immune responses to virus, bacteria and other antigens.}

An acute bout of exercise does not have a significant response to the immune consequences for health. A physically active lifestyle has shown to mitigate the changes associated with age, which take place in the adaptive cells of the immune system, but the mechanism of this concept is yet to be identified. PA might directly limit the escalation of memory $\mathrm{T}$ cells, wherein the memory $\mathrm{T}$ cells play a primary role in managing systemic inflammation. Restricting the increase in memory T cells indicates the fixed "size" of the immune system, the limited potential to generate antigennaïve $\mathrm{T}$ cells, and these limitations cause a decline in the immunity in the elderly. Nevertheless, exercise can indirectly influence the accumulation of memory $\mathrm{T}$ cells by decreasing viral reactivation, or preventing $\mathrm{T}$ cell senescence, by regulating deposition and dysfunction of adipose tissue, which causes inflammation and oxidative stress with ageing and obesity. Therefore, PA and regular exercise are encouraged, especially for elderly who tend to be at the highest risk of infections and those who may benefit the most from the exercise-induced immune competency.(14) Since both influenza and coronaviruses lead to respiratory tract infections, they can cause morbidity and mortality, particularly in the immune-compromised individuals and those who are susceptible to viruses.(15) Normally, moderateintensity exercises can be performed by individuals with mild upper respiratory tract symptoms, such as the runny nose, sinus congestion, mild sore throat, etc. However, if an individual experiences severe sore throat, shortness of breath, body aches, general fatigue, chest cough, or fever, he must avoid exercises and seek medical care.(15),(16)

\section{Effect of Obesity on the Body during Quarantine}

Reduced physical activity is common in obese individuals as compared to the lean ones. A decrease in PA due to self or insulin resistance in obese individuals alters the immune response against microbial agents by macrophage activation and/or inhibition of pro-inflammatory cytokines.(17) Obese individuals have a modified innate and adaptive immune response, marked by a chronic and low-grade inflammatory state. Studies show that obesity tends to inhibit virus-specific CD8 $+\mathrm{T}$ cell responses as well as antibody responses to the seasonal influenza vaccine, the reason being the suboptimal macrophage functionality and maturation in them.(17) Studies have demonstrated that chronic inflammation associated with obesity induces reduced activation of the macrophage upon introduction of an antigen, which further leads to dampened production of pro-inflammatory cytokines.(17) This results in poor vaccination effectiveness in obese subjects, and this describes the presence of antiviral-resistant and vaccine-free variants in obese individuals. There is also a reduction in $\mathrm{B}$ and $\mathrm{T}$ cell responses, resulting in increased susceptibility, and delay in the resolution of the viral infection. (17) Obese patients may demonstrate an alteration in the number and function of lymphocytes, resulting in altered memory $\mathrm{T}$ cell responses and effectiveness of the vaccine. A robust response of CD8 $+\mathrm{T}$ cells plays a key role in defending against new influenza virus strains and subtypes.

\section{Factors Which Make Obese Individuals More Contagious} Than Leans

1. Obese individuals with influenza spread the virus for prolonged periods (up to $104 \%$ longer) as compared to the lean individuals, potentially raising the chances of spreading the virus to other individuals.(18) Since the incubation time for Covid-19 is a forethought 14 days and obese subjects exhibit prolonged shedding of the virus, quarantine must be extended in obese compared to lean subjects. $(17,18)$

2. The microenvironment in obese individuals supports the rise of more virulent strains of viruses primarily due to their delayed and decreased potential to produce interferons. (19)

3. Body mass index (BMI) shows a positive correlation with the infectious virus in the exhaled air. $(17,20)$

PA has an immune-modulatory action, which focuses on enhancing weight loss and immune modulation. Both mortality and morbidity can be dampened with routine exercise, although weight loss might not be significant. Regular PA produces positive effects on metabolic health, such as in obesity, diabetes mellitus, and metabolic syndrome, and immunological health by enhancing immune activation levels, improving vaccination efficacy and controlling immune senescence. Several studies have demonstrated that routine PA and exercises increase the levels of TLR (toll-like receptor)-mediated cytokine production during microbial infection, improving the resistance of the host to pathogen invasion.(17) Besides, exercise strengthens the antioxidant defence mechanism and also reduces oxidative stress. Regular PA emerges as a cornerstone as a precautionary measure against influenza viral infection and other metabolic diseases in obese subjects to improve the host defence. In the elderly, the interventions used in PA have shown the ability to decrease the chances of complications by regulating inflammation, enhancing the immune system and improving vaccination outcomes.(17),(18) 


\begin{tabular}{|c|c|c|c|c|}
\hline Sl. No. & Exercises & Description & Duration/ Repetition & Advantage \\
\hline 1 & Knee to elbow & Bend one of your knees and touch it with the opposite elbow, alternatively. & $\begin{array}{l}\text { Do the exercise for } 1 \text { to } 2 \text { minutes, rest for } 30 \\
\text { to } 60 \text { seconds, and repeat up to } 5 \text { times. }\end{array}$ & $\begin{array}{l}\text { Enhances heart } \\
\text { and breathing } \\
\text { rates. }\end{array}$ \\
\hline 2 & Plank & $\begin{array}{l}\text { Keeping the hips at the level of the head and elbows under the shoulders, support your } \\
\text { forearms firmly on the ground. }\end{array}$ & $\begin{array}{l}\text { Hold for } 20 \text { seconds or more, and rest for } 30 \\
\text { to } 60 \text { seconds }\end{array}$ & $\begin{array}{l}\text { Strengthens belly, } \\
\text { arms and legs. }\end{array}$ \\
\hline 3 & $\begin{array}{c}\text { Back } \\
\text { extensions }\end{array}$ & $\begin{array}{l}\text { On a mat, lie on your stomach and straighten your legs behind you. With the elbows on } \\
\text { the ground and shoulders down, lift your upper back while maintaining your head and } \\
\text { neck in neutral, and pressing your hips into the mat. }\end{array}$ & $\begin{array}{l}\text { Do the exercise } 10 \text { times or more, rest for } 30 \\
\text { to } 60 \text { seconds, and repeat up to } 5 \text { times. }\end{array}$ & $\begin{array}{l}\text { Strengthens back } \\
\text { muscles. }\end{array}$ \\
\hline 4 & Squats & $\begin{array}{l}\text { Keeping the feet at a hip distance with the toes pointing outwards, bend the knees to a } \\
\text { comfortable range with the heels on the ground and the knees over the feet. Bend and } \\
\text { stretch the legs. }\end{array}$ & $\begin{array}{l}\text { Do this exercise } 10 \text { times or more, rest for } 30 \\
\text { to } 60 \text { seconds, and repeat up to } 5 \text { times. }\end{array}$ & $\begin{array}{l}\text { Strengthens legs } \\
\text { and glutes. }\end{array}$ \\
\hline 5 & Side knee lifts & Touch your knee with your elbow, lifting the knee to the side, alternatively. & $\begin{array}{l}\text { Do this for } 1 \text { to } 2 \text { minutes, rest for } 30 \text { to } 60 \\
\text { seconds, and repeat up to } 5 \text { times. }\end{array}$ & $\begin{array}{l}\text { Enhances heart and } \\
\text { breathing rates }\end{array}$ \\
\hline 6 & Superman & $\begin{array}{l}\text { In a quadruped position, with your hands under your shoulders, and knees under your } \\
\text { hips, lift one arm forward and the opposite leg back, alternatively. }\end{array}$ & $\begin{array}{l}\text { Do this exercise } 20 \text { times or more, rest for } 30 \\
\text { to } 60 \text { seconds, and repeat up to } 5 \text { times. }\end{array}$ & $\begin{array}{l}\text { Strengthens belly, } \\
\text { glutes and back } \\
\text { muscles. }\end{array}$ \\
\hline 7 & Bridge & $\begin{array}{l}\text { Lie on your back, then put your feet on the ground with the knees over the heels. Lift the } \\
\text { hips to your comfortable range and slowly lower them again. }\end{array}$ & $\begin{array}{l}\text { Do this exercise } 10 \text { times or more, rest for } 30 \\
\text { to } 60 \text { seconds, and repeat up to } 5 \text { times. }\end{array}$ & $\begin{array}{l}\text { Strengthens } \\
\text { glutes. }\end{array}$ \\
\hline 8 & Chair dips & $\begin{array}{l}\text { Hold onto the seat of a chair, with your feet about half a meter away from the chair. Bend } \\
\text { your arms as you lower your hips to the ground, and then straighten the arms. }\end{array}$ & $\begin{array}{l}\text { Do this exercise } 10 \text { times or more, rest for } 30 \\
\text { to } 60 \text { seconds, and repeat up to } 5 \text { times. }\end{array}$ & Strengthens triceps. \\
\hline 10 & Child's pose & $\begin{array}{l}\text { With the knees on the ground, bring your hips to your heels. Rest your belly on your } \\
\text { thighs and actively stretch your arms forward. Breathe normally. }\end{array}$ & Hold this position for 20 seconds or more. & $\begin{array}{l}\text { Stretching of back, } \\
\text { shoulders and sides of } \\
\text { the body. }\end{array}$ \\
\hline 11 & $\begin{array}{c}\text { Seated } \\
\text { meditation }\end{array}$ & $\begin{array}{l}\text { Sit comfortably on the floor with your legs crossed or sit on a chair. Keep your back is } \\
\text { straight. Close your eyes, relax your body and progressively deepen your breathing. }\end{array}$ & $\begin{array}{c}\text { Remain in this position for } 5 \text { minutes or } \\
\text { more. }\end{array}$ & Relaxation. \\
\hline 12 & $\begin{array}{l}\text { Legs up the } \\
\text { wall }\end{array}$ & $\begin{array}{l}\text { Bring your hips to close }(5 \text { to } 10 \mathrm{~cm}) \text { to the wall and let your legs rest. Close your eyes, } \\
\text { relax your body and progressively deepen your breathing. Concentrate on your breath, } \\
\text { trying not to focus on any thought or concern. }\end{array}$ & Rest in this pose for up to 5 minutes. & $\begin{array}{l}\text { Relaxation and de- } \\
\text { stressing. }\end{array}$ \\
\hline \multicolumn{5}{|c|}{ Table 1. Home-Based Exercises Prepared by WHO, Europe to Support Individuals in Staying Physically Active in Quarantine. (4) } \\
\hline
\end{tabular}

\section{Relationship between Physical Health and Immune System}

Previous studies have demonstrated that a physically active lifestyle dampens the risk of developing infections.(14) During intense aerobic exercise, peripheral count of lymphocytes in the blood significantly heightens, leading to the idea that PA and exercise play an important role to enhance the immune system. This is attributed to high blood pressure and shear movements during exercise. Furthermore, due to the release of adrenaline during exercise, the primary controller is the adrenergic stimulation of beta-2-adrenergic receptors on the lymphocyte surface. This leads to endothelial detachment and consequent lymphocyte recirculation into the bloodstream.(14) According to the theory of acute stress/immune enhancement exercise, these immune cells supposedly recognize and kill cells, which are pathogeninfected or have become weakened or malignant. Evidence from several studies has indicated that PA can be beneficial in enhancing the poor immunological responses in the elderly.(14) Besides, preliminary evidence shows that PA and routine exercise can also restrict or postpone the weakening of the immune system. Therefore, exercise must be encouraged, particularly for those at the highest risk of infection, and who can benefit the most from the exerciseinduced immune competency. Tissue macrophage antipathogenic activity occurs throughout aerobic exercise in conjunction with increased immunoglobulin recirculation, anti-inflammatory cytokines, neutrophils, natural killer cells, cytotoxic T cells, too immature B cells, all of which play vital roles in immune defense function and metabolic safety.(21) Injury and inflammation of the muscle tissue caused by the exercise evoke a robust innate immune response comprising granulocytes, monocytes and macrophages. To modulate the innate immune response, immune-specific proteins are generated, which includes oxylipins to initiate, mediate and resolve this process.(21). The intense, exercise-induced perturbation in metabolites, lipid mediators, and proteins has an impact on the immune function, decreasing the ability of immune cells to enhance levels of oxygen consumption after activation. Oxygen and biosynthetic demands are associated with the activation of the immune system, and the immune cells need metabolic reprogramming to generate sufficient energy to meet those demands. PA facilitates immunosurveillance, and daily PA provides various health benefits that include reduced disease incidence and decreased systemic inflammation. Several epidemiological studies revealed that routine PA correlates with lower rates of death and incidence of infection.(21) Furthermore, there is evidence of enhanced antibody responses to influenza immunization in individuals involved in regular exercise training schemes. Primarily, exercise stimulates the recirculation of chief immune cells, and an anti-inflammatory and antioxidant environment intervenes via different pathways. There is ample evidence to suggest that older adults are more prone to infection, and have weaker immune responses for vaccines relative to their younger counterparts. ${ }^{(12)}$

\section{Considerations for Physical Activities}

It is important to note that before carrying out any physical activity, the relationship between the cost $\mathrm{x}$ benefit of it needs to be a highlight. Besides, we must avoid osteoarticular damage, as well as maximizing the effectiveness of the chosen model will be essential for more effective training that generates adequate benefits for the performer.(3) It is important to select the exercise of correct intensity, keeping in mind the health status and fitness level. While performing any light and moderate-intensity physical activities, one must breathe comfortably and hold a conversation. In case a 
person is not regularly active, he should start with lowintensity activities, like walking and low impact exercises, and gradually increase the intensity over a few weeks. If a person is presenting with fever, cough and difficulty breathing, exercises should be ceased immediately.(1) Some home-based exercises prepared by WHO (Europe) to promote physical activities in quarantine are tabulated in Table 1.

\section{Principal Elements to be Considered while Designing an Exercise Program}

1. Type of exercise: A multidimensional exercise program is a preferable form of exercise routine comprising of aerobic, coordination, balance, resistance and mobility training exercises. Recent researches suggest that the exercise training session should also combine the concept of cognitive training.

2. Frequency of exercise: Normally, the recommended frequency of exercise per week is 5 days. However, in the current situation of quarantine, it can be raised to 5-7 days per week along with adaptations in exercise volume and exercise intensity.

3. Volume of Exercise: Normally, the minimum recommended volume of exercise per week is 150 to 300 min of aerobic exercise and 2 resistance training sessions. However, during current quarantine, the volume of aerobic exercise can be increased to 200-400 min per week allocated among 5-7 days so to counterbalance for the decreased normal daily PA levels. Furthermore, resistance exercise of at least 2- 3 days per week is suggested. (11)

4. Intensity of exercise: Normally, the recommended intensity of exercise per week is moderate along with some amount of vigorous exercise. Exercise at moderate intensity has shown to enhance the immune system; however vigorous intensity may tend to inhibit it, especially in the elderly. Thus, moderate-intensity exercises $(40-60 \%$ heart rate reserve or $65-75 \%$ of maximal heart rate) are preferred to enhance the protective role of exercise during the quarantine.(11)

\section{Types of Exercises Recommended during Quarantine}

1. Aerobic exercise: In the absence of infection, aerobic exercises are recommended to strengthen the cardiovascular health while in the presence of infection, during the period of mild symptoms, moderate daily aerobic exercise can improve lung ventilation and benefit immune function. Jumping jacks, jogging in place, or dancing can be performed in small spaces, making them reliable forms of aerobic exercises. It is advised to keep the room ventilated as proper ventilation allows any viral particles present in the air to exit the room and oxygenates the room, which is helpful for the lungs and all-around health.(16)

2. Deep breathing: Performing deep breathing exercises improves lung capacity. Expelling viral particles from the more stagnant areas of the lung may further decrease self-exposure to viral particles. Deep breathing is often recommended for health and wellbeing and can be done multiple times a day on a regular schedule.(16)
3. High-intensity interval training (HIIT): HIIT Body work is an intense interval training that seeks to work in a short time improving the metabolic and neuromuscular abilities of the practitioner. Most of the time, short intervals of high-intensity exercise $(20,30$ seconds) are used, interspersed with brief rest intervals.(3) HIIT Body Work doesn't require any additional devices and increases the maximum oxygen capacity. However, this method, in most cases, requires sudden movements of jumps and changes in the centre of gravity with speed, which can, in general, compromise the soft tissues (ligaments and tendons), thus should be performed in the presence of experts only.

4. Manual Resistance Training: In manual resistance training, the resistance is given by a training partner to generate an external overload for carrying out concentric and eccentric muscle actions. It does not require additional devices. Because of its easy applicability for individuals with different levels of physical fitness, the adequate control of movements and the quick adjustment in the load $\mathrm{x}$ speed ratio (performed by the companion).

5. Self-resisting exercise: These are carried out using an external resistance caused by the performer. Researches demonstrate effective muscle activation during selfresisting movements. However, the effectiveness of the resistance imposed on this model does not remain constant during the protocol, due to the fatigue generated.(3)

6. Training with elastic resistance: It is carried out in two ways, hybrid elastic resistance, and simple elastic resistance. In hybrid elastic resistance, the elastic resistance (bands or tubes) is used together with traditional weights, commonly used among sportspersons for better development of power, acceleration and movement speed in athletes. In simple elastic resistance, only elastic bands and elastic tubes are used and the resistance is given by the intensity of the band or tube.(6) It shows good results in young adults as well as the elderly through the positive changes in musculoskeletal health, such as muscle hypertrophy, decreased body fat and increased muscle strength and physical performance. Due to its ease of use in different spaces, for the most varied audiences, security and, the possibility of control, elastic resistance training has a high potential for application in domestic environments, and can considerably help the population that is quarantined. $(3,6)$

\section{CONCLUSIONS}

To minimize the effects of Covid-19, health organizations advise social isolation in the form of quarantine, which led to reduced regular physical activity and exercise leading to a further compromise in the physical health and immunity of individuals. Physical activity and exercise are essential during quarantine to retain the normal physiological function and to combat the mental and physical impacts of the Covid-19 pandemic. Self-quarantine can also be challenging to the mental health of people, which can be mitigated by regular exercises and relaxation techniques. Furthermore, physical 
activity and exercise have shown to reduce the chances of developing opportunistic infections, and tend to improve the body's natural immune responses to these infectious agents. Also, obese individuals with influenza spread the virus for prolonged periods than lean individuals, their microenvironment supports the rise of more virulent strains of the virus, and their high BMI has a positive correlation with the infectious virus in the exhaled air. Normally, moderateintensity exercises are advised in individuals with mild upper respiratory tract symptoms, such as the runny nose, sinus congestion, mild sore throat, etc., but if an individual presents with a severe sore throat, cough, shortness of breath, body aches, general fatigue, or fever, he must avoid exercises and seek medical care. Lastly, the principal elements to be considered while designing an exercise program include the type of exercise, frequency of exercise, volume of exercise and intensity of exercise.

\section{REFERENCES}

[1] Be active during Covid-19. World Health Organization 2020. https://www.who.int/news-room/q-a-detail/beactive-during-covid-19

[2] Singhal T. A review of coronavirus disease-2019 (Covid19). Indian J Pediatr 2020;87(4):281-6.

[3] Miguel H, Campos MVA. The use of different models of physical exercises performed at home during the Covid19 pandemic: positive and negative points. InterAm J Med Health 2020;3:e202003005.

[4] Stay physically active during self-quarantine. World Health Organization http://www.euro.who.int/en/health-topics/healthemergencies/coronavirus-covid-19/novel-coronavirus2019-ncov-technical-guidance/stay-physically-activeduring-self-quarantine

[5] Kim SW, Su KP. Using psychoneuroimmunity against Covid-19. Brain Behav Immun 2020;S08891591(20)30391-3.

[6] Campos MVA, Miguel H. Elastic resistance training: resistance exercise alternative in the home environment during Covid-19 pandemic. InterAm J Med Health 2020;3:e202003006.

[7] Hall G, Laddu DR, Phillips SA, et al. A tale of two pandemics: how will Covid-19 and global trends in physical inactivity and sedentary behavior affect one another? Prog Cardiovasc Dis 2020;00330620(20)30077-3.
[8] Sharan D, Mohandoss M, Ranganathan R, et al Musculoskeletal disorders of the upper extremities due to extensive usage of hand held devices. Ann of Occup and Environ Med 2014;26:22.

[9] Lee M, Hong Y, Lee S, et al. The effects of smartphone use on upper extremity muscle activity and pain threshold. J Phys Ther Sci 2015;27(6):1743-5.

[10] Berolo S, Wells RP, Amick BC. Musculoskeletal symptoms among mobile hand-held device users and their relationship to device use: a preliminary study in a Canadian university population. Appl Ergon 2011;42(2):371-8.

[11] Jiménez-Pavón D, Carbonell-Baeza A, Lavie CJ. Physical exercise as therapy to fight against the mental and physical consequences of Covid-19 quarantine: special focus in older people. Prog Cardiovasc Dis 2020;S00330620(20)30063-3.

[12] Song Y, Ren F, Sun D, et al. Benefits of exercise on influenza or pneumonia in older adults: a systematic review. Int J Environ Res Public Health 2020;17(8):2655.

[13] Suchomel TJ, Nimphius S, Bellon CR, et al. The importance of muscular strength: training considerations. Sports Med 2018;48(4):765-85.

[14] Campbell JP, Turner JE. Debunking the myth of exerciseinduced immune suppression: redefining the impact of exercise on immunological health across the lifespan. Front Immunol 2018;9:648.

[15] Zhu W. Should, and how can, exercise be done during a coronavirus outbreak? An interview with Dr. Jeffrey A. Woods. J Sport Health Sci 2020;9(2):105-7.

[16] Elias B, Shen C, Bar-Yam Y. Respiratory health for better Covid-19 outcomes. New England Complex Systems Institute March 16, 2020.

[17] Luzi L, Radaelli MG. Influenza and obesity: its odd relationship and the lessons for Covid-19 pandemic. Acta Diabetol 2020;57(6):759-64.

[18] Maier HE, Lopez R, Sanchez N, et al. Obesity increases the duration of influenza a virus shedding in adults. J Infect Dis 2018;218(9):1378-82.

[19] Klinkhammer J, Schnepf D, Ye L, et al. IFN- $\lambda$ prevents influenza virus spread from the upper airways to the lungs and limits virus transmission. Elife 2018;7:e33354.

[20] Yan J, Grantham M, Pantelic J, et al. Infectious virus in exhaled breath of symptomatic seasonal influenza cases from a college community. Proc Natl Acad Sci USA 2018;115(5):1081-6.

[21] Nieman DC, Wentz LM. The compelling link between physical activity and the body's defense system. Journal of Sport and Health Science 2019;8(3):201-17. 